\title{
Multi Modal Dynamic Linear Viscoelastic Back Analysis for Asphalt Mixes
}

\author{
Jean-Claude Carret ${ }^{1} \mathbb{D} \cdot$ Herve Di Benedetto $^{1} \cdot$ Cedric Sauzeat $^{1}$
}

\begin{abstract}
In this paper, an investigation was performed to determine the accuracy of a simplified viscoelastic back analysis to interpret dynamic loading tests on asphalt mixes (AM). First, quasi-static cyclic tension-compression lab tests were performed on different AM to fit the 3 dimensional 2S2P1D linear viscoelastic (LVE) model. Considering these tests on very different types of AM, a LVE material with "averaged" viscoelastic properties was obtained. Then, these "averaged" viscoelastic properties were considered to perform finite elements method numerical simulations of dynamic loading tests on a cylinder. The simulations were performed at ten different temperatures from -40 to $50{ }^{\circ} \mathrm{C}$. The longitudinal, flexural and torsional modes of vibration are studied. The complex Young's modulus and complex Poisson's ratio were first obtained using the viscoelastic 2S2P1D model at the first resonance frequency for the three studied modes of vibration. Then, a combined viscoelastic back analysis, which has the advantage of simplicity, was used to determine the elastic equivalent properties and the phase angle of the material. The results obtained directly with the 2S2P1D model and the results from the combined viscoelastic back analysis results regarding both the Young's modulus and the Poisson's ratio are discussed in the paper.
\end{abstract}

Keywords Asphalt mixes · Viscoelasticity $\cdot$ Dynamic loading $\cdot$ Finite element calculation $\cdot$ Back analysis

\section{Introduction}

Seismic measurements such as impact loadings are economical, simple to perform and are nondestructive tests. These tests seem to be a good approach for providing accurate characterization of materials properties [1]. Measurement of the flying time in wave propagation tests [2-4] and measurement of the fundamental resonance frequencies through resonance testing $[5,6]$ have been used to determine the complex modulus of asphalt mixtures. These methods give information on the tested LVE material only for a limited number of resonance frequencies (1-3), which are, a priori, unknown. The analysis of the results of these tests is based on simplified approximate formulations used to determine a complex mod-

Jean-Claude Carret

jean-claude.carret@entpe.fr

Herve Di Benedetto

herve.dibenedetto@entpe.fr

Cedric Sauzeat

cedric.sauzeat@entpe.fr

1 LTDS (UMR CNRS 5513), University of Lyon / ENTPE, Rue M. Audin, 69518 Vaulx en Velin, France ulus for each considered temperature and mode of vibration $[7,8]$. Ryden and Gudmarsson $[9,10]$ applied resonant acoustic spectroscopy (RAS) to calculate resonance frequencies of cylindrical discs and beams for different modes of vibration. These tests require simplified method such as the half-power bandwidth method to evaluate the damping of the specimen. In this paper, a comparison between the direct viscoelastic analysis and a simplified back analysis of a specimen under impact loadings were performed. The differences between the two approaches and the limitations of the simplified back analysis were highlighted. LVE finite element method numerical simulations of impact loadings on a cylindrical specimen were carried out. These numerical tests were performed at 10 different temperatures between -40 and $50{ }^{\circ} \mathrm{C}$ for three different modes of vibration: the longitudinal mode, the flexural mode and the torsional mode. The analysis of these computations were limited to the first resonance frequency for each mode. Viscoelastic properties of the specimen were estimated at the corresponding resonance frequency for each mode of vibration and for each temperature using the 2S2P1D linear viscoelastic model. Then, a combined back analysis-it is the type of method mostly used to analyze impact testing on asphalt materials - was used to evaluate the elastic equiva- 
Fig. 1 (left)

Tension-compression test apparatus used for tests on asphalt mixes; (right) detailed scheme of measurement devices and sample
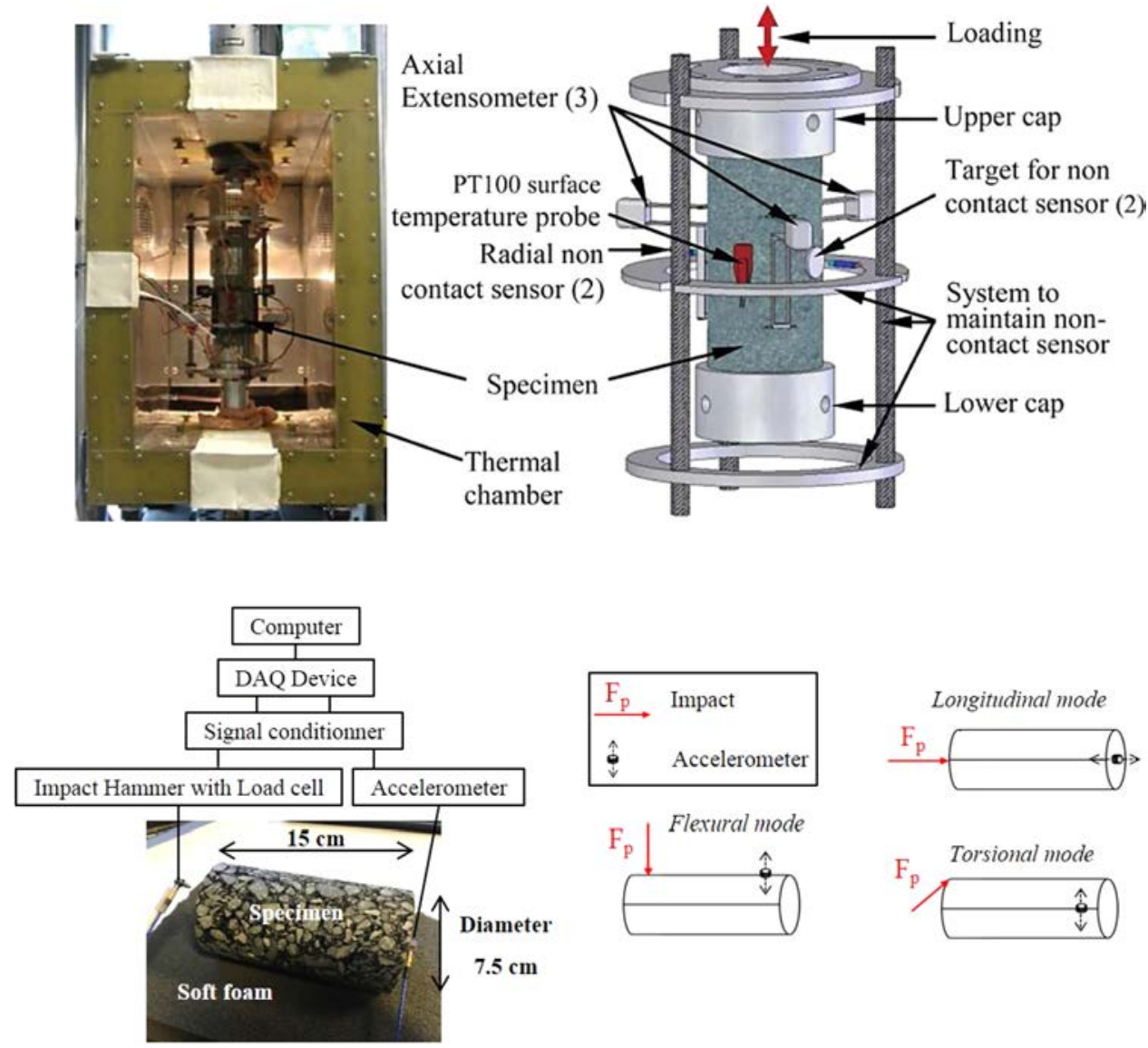

Fig. 2 (left) Dynamic impact tests set-up (ENTPE/LTDS laboratory), case of the longitudinal mode; (right) Position of the accelerometer and of the impact for the three considered modes of vibration lent properties and the phase angle of the material. Finally, results obtained directly with the 2S2P1D model and from the combined back analysis were compared.

\section{Materials and Experimental Tests}

\subsection{Materials and Considered Material for Modelling}

38 specimens are first considered in this study. They were previously tested (cyclic tension-compression test-see following section-) during 4 different $\mathrm{Ph}$.D. theses performed at the University of Lyon / ENTPE, LTDS laboratory [11-17]. The wide variety of these specimens represents a good range of asphalt mixes types and makes it possible to consider a material with averaged viscoelastic properties. This material was given the average values of the constants of the 2S2P1D LVE model obtained for the 38 specimens. All the numerical simulations and analyses that are presented in this paper were performed on this average material.

\subsection{Cyclic Tension-Compression Tests}

The tension-compression tests were used to determine the complex Young's modulus and the complex Poisson's ratio of asphalt mixes. The tests were performed on cylindrical sam- ples (150 mm high with a $75 \mathrm{~mm}$ diameter). Cyclic sinusoidal loadings were applied using a hydraulic press, as shown in Fig. 1, in strain-controlled mode with an amplitude of around $5 * 10^{-5} \mathrm{~m} / \mathrm{m}$.

The axial stress $\left(\sigma_{1}=\sigma_{0} \cdot \sin \left(\omega t-\phi_{E}\right)\right)$ was measured with a load cell with a $\pm 25 \mathrm{kN}$ maximum load and a $25 \mathrm{~N}$ accuracy. The axial strain $\left(\varepsilon_{1}=\varepsilon_{01} \cdot \sin (\omega t)\right)$ was obtained by means of three extensometers placed at $120^{\circ}$ from each other (Fig. 1). The radial strain $\left(\varepsilon_{2}=\varepsilon_{02} \cdot \sin \left(\omega t+\phi_{\nu}\right)\right)$ was deduced from measurements of two non-contact transducers (Fig. 1). The complex modulus and the complex Poisson's ratio at different loading frequencies (from 0.01 to $10 \mathrm{~Hz}$ ) and different temperatures (from -30 to $50{ }^{\circ} \mathrm{C}$ ) are calculated according to Eqs. 1 and 2 where $\phi_{E}$ and $\phi_{\nu}$ are the phases of the complex modulus and of the complex Poisson's ratio respectively.

$$
\begin{aligned}
& E^{*}(\omega)=\frac{\sigma_{1}^{*}}{\varepsilon_{1}^{*}}=\left|E^{*}(\omega)\right| e^{i \Phi_{E}} \\
& \nu^{*}(\omega)=-\frac{\varepsilon_{2}^{*}}{\varepsilon_{1}^{*}}=\left|\nu^{*}(\omega)\right| e^{i \Phi_{v}}
\end{aligned}
$$




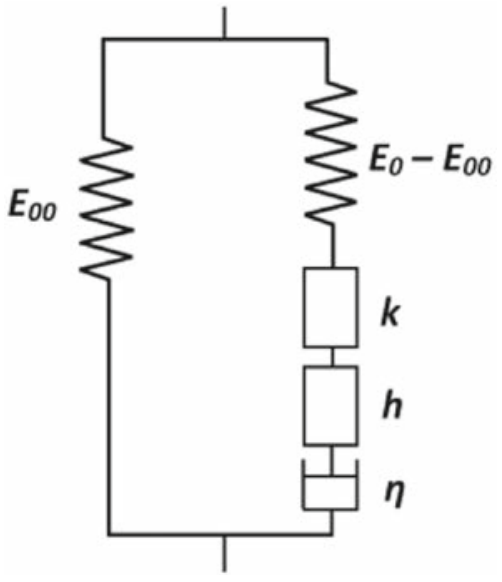

Fig. 3 Representation of the 2S2P1D LVE model in the 1-dimension case

\subsection{Dynamic Impact Tests and Conditions for the Numerical Simulations}

The sample geometry used for tension-compression test (7.5 $\mathrm{cm}$ diameter and $15 \mathrm{~cm}$ height) was considered for dynamic test. In this paper only numerical tests are presented but the associated lab test set-up of the dynamic impact tests is shown on the left of Fig. 2. The loading is manually applied on the specimen with an impact hammer equipped with a load cell (PCB model 086E80). The standing wave modes are measured with an accelerometer (PCB model 353B15) glued to the specimen. During the test, the specimen lays on soft foam to ensure free boundary conditions [6]. The impact hammer and the accelerometer are connected to the data acquisition system that consists of a signal conditioner (PCB model 482C15), a data acquisition device (NI DAQ USB-6251) and a computer (see Fig. 2). It is possible to excite different modes of vibration depending on the position of the impact and of the accelerometer [18,19]. The configurations for the three different modes of vibration considered in this study are described on the right of Fig. 2. These configurations were used for the FEM numerical simulations (see Sect. 3.2).

\section{Linear Viscoelastic Analysis}

\subsection{S2P1D LVE Isotropic Model}

The tension-compression tests results were analyzed using the 2S2P1D LVE model developed at ENTPE [20-22]. It consists of a combination of two springs, two parabolic creep elements (also called fractional-derivative model [23]) and one dashpot as schematized in the 1-dimension case in Fig. 3.
The introduced model has seven parameters and the complex modulus of the 2S2P1D model is expressed according to Eq. 3,

$$
\begin{aligned}
& E_{2 S 2 P 1 D}^{*}(\omega) \\
& =E_{00}+\frac{E_{0}-E_{00}}{1+\delta(j \omega \tau)^{-k}+(j \omega \tau)^{-h}+(j \omega \beta \tau)^{-1}}
\end{aligned}
$$

where $j$ is complex number defined by $j^{2}=-1, \omega$ is the angular frequency ( $\omega=2 \pi f$, where $f$ is the frequency), $E_{00}$ is the static modulus when $\omega \rightarrow 0, E_{0}$ is the glassy modulus when $\omega \rightarrow+\infty, \delta$ is a dimensionless constant, $k$ and $h$ are dimensionless exponents such as $0<k<h<1, \beta$ is a dimensionless constant related to the Newtonian viscosity $\eta$ by $\eta=\left(E_{0}-E_{00}\right) \beta \tau$ and $\tau$ is the characteristic time, function of temperature. The time-temperature superposition principle (TTSP) is verified for asphalt mixes in the linear and nonlinear domains $[16,24,25]$ using:

$\tau(T)=a_{T}(T) \tau_{r e f}$

where $a_{T}$ is the shift factor at the temperature T defined by the Williams-Landel-Ferry (WLF) equation [26]:

$\log \left(a_{T}\right)=-\frac{C_{1}\left(T-T_{r e f}\right)}{C_{2}+T-T_{r e f}}$

where $C_{1}$ and $C_{2}$ are the two constants of the WLF equation and $T_{r e f}$ is the reference temperature. The WLF parameters used in this study are listed in Table 1 and the corresponding shift factors are plotted in Fig. 4. Di Benedetto et al. [27] extended the 2S2P1D model to characterize the complex Poisson's ratio as given in Eq. 6,

$$
\begin{aligned}
& v_{2 S 2 P 1 D}^{*}(\omega) \\
& =v_{00}+\frac{v_{0}-v_{00}}{1+\delta\left(j \omega \tau_{v}\right)^{-k}+\left(j \omega \tau_{\nu}\right)^{-h}+\left(j \omega \beta \tau_{\nu}\right)^{-1}}
\end{aligned}
$$

where $v_{00}$ is the low frequency Poisson's ratio when $\omega \rightarrow 0$ and $v_{0}$ is the high frequency Poisson's ratio when $\omega \rightarrow+\infty$. The same values of the parameters $\delta, k, h$ and $\beta$ are used to determine both the complex modulus and complex Poisson's ratio while $\tau$ is determined also for the Poisson's ratio and is therefore labeled $\tau_{v}$. The constants of the 2S2P1D LVE model for the material used in the numerical simulations (see Sect. 2.1) are given in Table 1. The complex modulus ( $\left.\mathrm{E}^{*}\right)$ and the complex Poisson ratio $\left(v^{*}\right)$ of the average material are plotted in Fig. 5 (master curves) and Fig. 6 (normalized cole-cole curves).

\subsection{Finite Element Method Simulations}

Finite element method is widely used on pavement for different applications [28-32]. In this study, the behavior of 
Table 1 2S2P1D constants at the reference temperature of $10{ }^{\circ} \mathrm{C}$ for the material with "averaged" viscoelastic properties of asphalt mixes used for the numerical simulations

\begin{tabular}{|c|c|c|c|c|c|c|c|c|c|c|c|}
\hline$E_{00}(M P a)$ & $E_{0}(M P a)$ & $v_{0}$ & $v_{00}$ & $\delta$ & $k$ & $h$ & $\beta$ & $\tau_{10^{\circ} C}(s)$ & $\tau_{v}(s)$ & $C_{1}$ & $C_{2}$ \\
\hline 105 & 35,000 & 0.19 & 0.42 & 2.15 & 0.17 & 0.525 & 505 & 0.1 & 3.165 & 30 & 210 \\
\hline
\end{tabular}

Fig. 4 Shift factor versus temperature for the considered WLF parameters
Fig. 5 Master curves at $10^{\circ} \mathrm{C}$ for the norm of the complex Young's modulus (solid line) and for the norm of the complex Poisson's ratio (dot line) for the material used for the numerical simulations
Fig. 6 Cole-Cole plot for the normalized complex Young's modulus and for the normalized complex Poisson's ratio for the material used for the numerical simulations
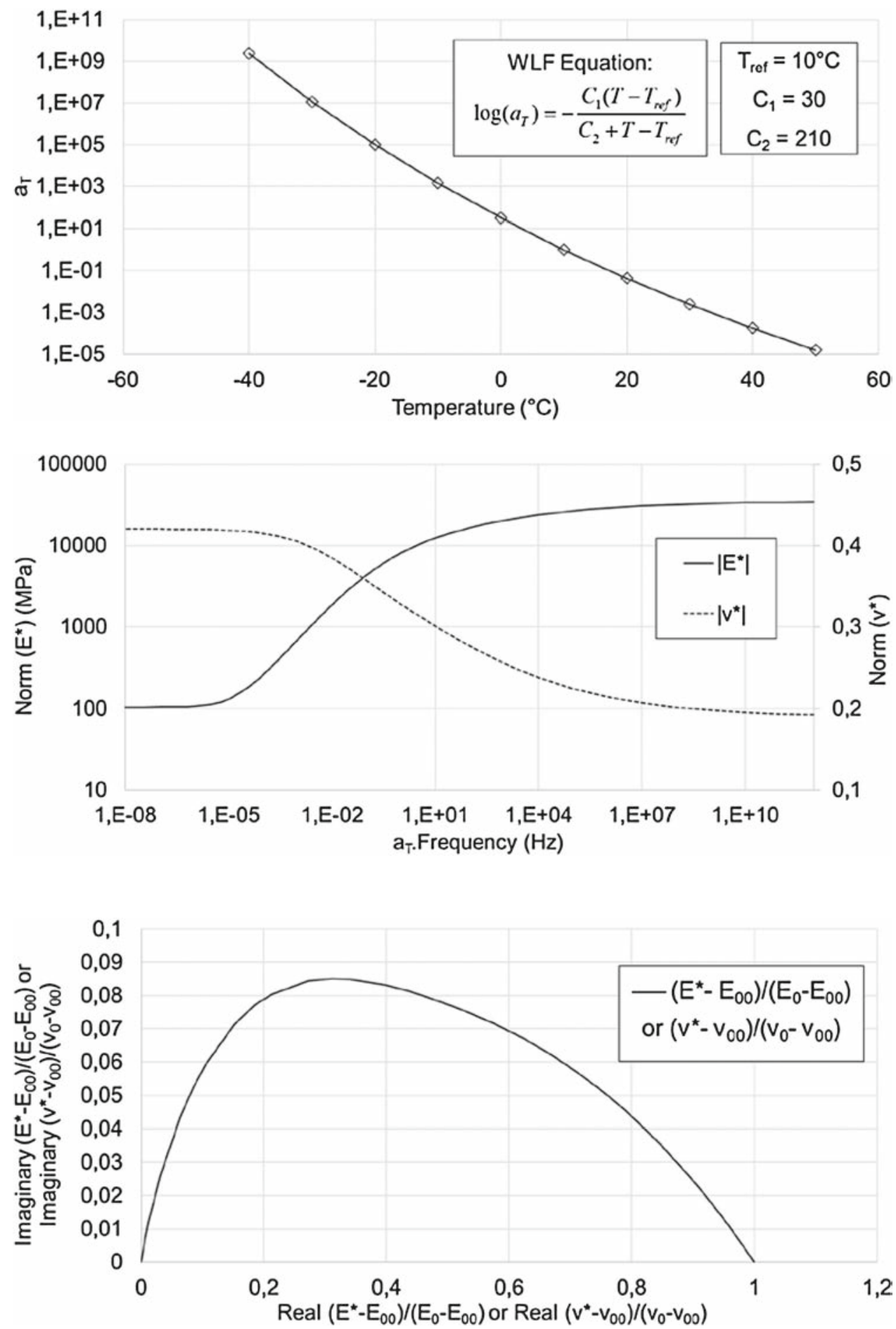
Fig. 7 FEM calculation and principle of the combined back analysis

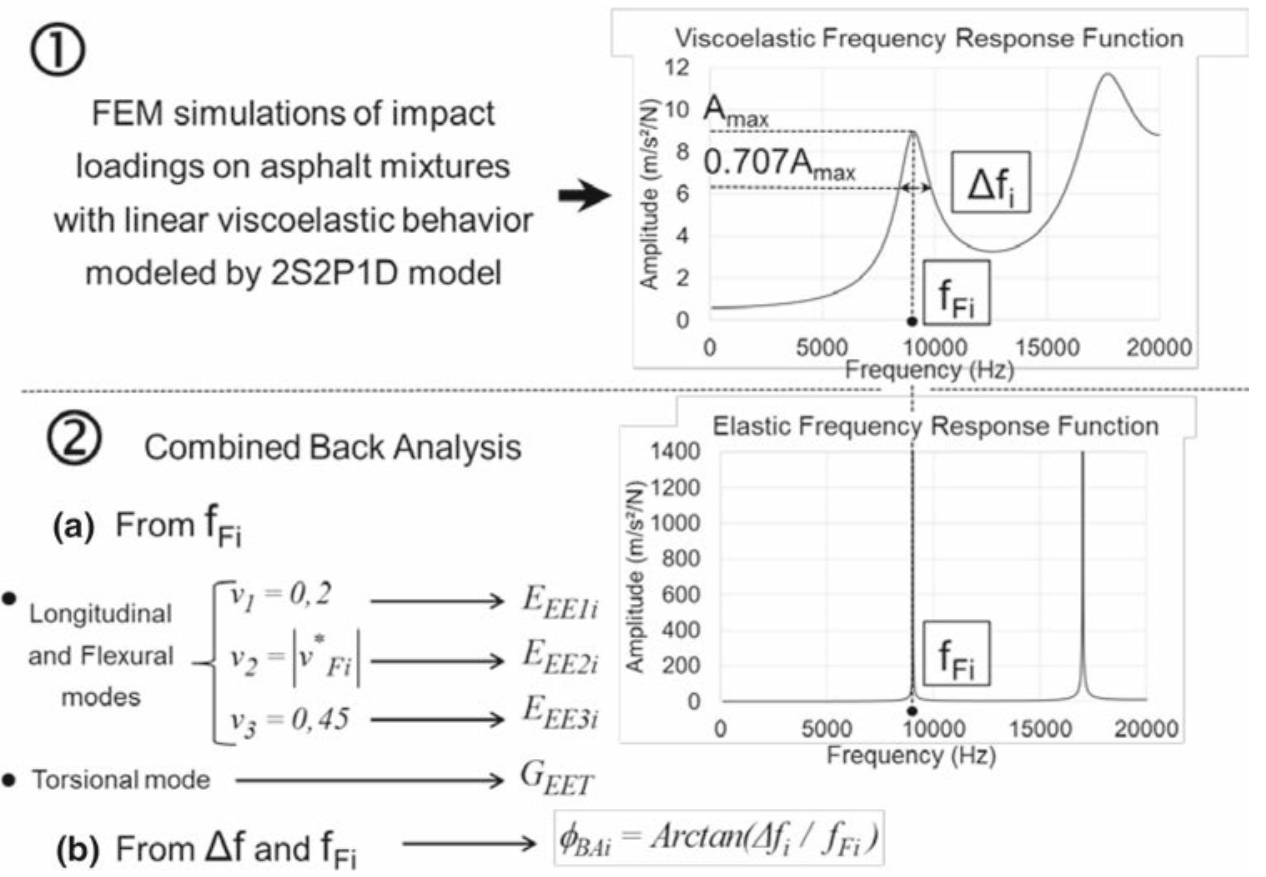

the material under dynamic loadings was calculated with FEM numerical simulations. The 2S2P1D model complex modulus and complex Poisson's ratio (Table 1) were used as input for the material properties and the material was considered as isotropic. The Comsol software was used to solve the three-dimensional equation of motion in the frequency domain (Eq. 7):

$-\rho \omega^{2} \mathbf{u}-\nabla \cdot \sigma=\mathbf{F}_{\mathbf{p}} e^{i \Phi}$

where $\omega$ is the angular frequency, $\rho$ is the density, $\mathbf{u}$ is the displacement vector, $\nabla$ is the gradient tensor operator, $i$ is complex number defined by $i^{2}=-1$ and $\sigma$ is the Cauchy stress tensor. $\mathbf{F}_{\mathbf{p}}$ is the unity load and $\phi$ is the phase of the cyclic load.

The unity load has been applied to the corresponding point of the hammer impact while the response has been determined at the position of the accelerometer for the three considered modes of vibration (see Fig. 2). The simulations were performed at 10 different temperatures every $10{ }^{\circ} \mathrm{C}$ from -40 to $50^{\circ} \mathrm{C}$, in a frequency range from 100 to 20,000 $\mathrm{Hz}$ considering steps of $20 \mathrm{~Hz}$. Finer analysis with steps of 1 $\mathrm{Hz}$ were also carried out around the first resonant frequency for each temperature and for each mode of vibration in order to obtain a better evaluation of the first resonance frequency and of the damping of the material. The geometry used in this study was a cylinder with a $75 \mathrm{~mm}$ diameter and a $150 \mathrm{~mm}$ height. The mesh consists of tetrahedral elements with a maximum element size of $2.5 \mathrm{~cm}$ that was determined through a convergence study.

\section{Combined Back Analysis}

Back analysis methods are used for different applications on pavement $[33,34]$. The proposed combined back analysis is based on the analysis of the first resonance peak for each temperature and for each mode of vibration. Figure 7 explains the principle of the back analysis for one temperature. In the first step (see Fig. 7), the first resonance frequency of each mode of vibration $f_{F i}$ ( $i=L$ for the longitudinal mode, $i=F$ for the flexural mode and $i=T$ for the torsional mode) was deduced from the FEM simulations. The frequency bandwidth $\Delta f_{i}$ was also determined for each mode of vibration from the FEM simulations using the half-power bandwidth method (see Fig. 3). For the longitudinal and flexural modes of vibration, the frequencies $f_{F i}$ were used in the 2S2P1D model to calculate the corresponding complex modulus $E_{F i}^{*}$, and Poisson's ratio, $v_{F i}^{*}$, according to Eqs. 8 and 9:

$$
\begin{aligned}
E_{F i}^{*} & =E_{2 S 2 P 1 D}^{*}\left(2 \pi f_{F i}\right) \\
v_{F i}^{*} & =v_{2 S 2 P 1 D}^{*}\left(2 \pi f_{F i}\right)
\end{aligned}
$$

For the torsional mode of vibration, the frequency $f_{F T}$ was used in the 2S2P1D model to calculate the corresponding complex shear modulus $G_{F T}^{*}$ according to Eq. 10:

$G_{F T}^{*}=G_{2 S 2 P 1 D}^{*}\left(2 \pi f_{F T}\right)$

The phase angle used in the back analysis $\phi_{B A i}$ was deduced using the following relationship suggested by Clough and Penzien [35]: 
$\phi_{B A i}=\operatorname{Arctan}\left(\frac{\Delta f_{i}}{f_{F i}}\right)$

In the second step of the combined back analysis (see Fig. 7), 2 different approaches were used. For the longitudinal and flexural modes of vibration, three cases corresponding to three different real values of Poisson's ratio of $0.2,\left|v_{F i}^{*}\right|$, and 0.45 were studied. Three elastic equivalent moduli $E_{E E 1 i}$, $E_{E E 2 i}$ and $E_{E E 3 i}$ associated to the three cases were backcalculated through elastic FEM numerical simulations for these two modes of vibration. For the torsional mode of vibration, the shear modulus is independent of the Poisson's ratio value. Therefore, only one elastic equivalent shear modulus $G_{E E T}$ was back-calculated through elastic FEM numerical simulations. Then $\phi_{B A i}$ was considered as the corresponding phase angle for the three considered modes of vibration. Three complex moduli $E_{B A 1 i}^{*}, E_{B A 2 i}^{*}$ and $E_{B A 3 i}^{*}$ were determined according to Eqs. 12-14 for the longitudinal and flexural mode and one complex shear modulus $G_{B A T}^{*}$ was determined according to Eq. 15.

$$
\begin{aligned}
E_{B A 1 i}^{*} & =E_{E E 1 i} e^{i \phi_{B A i}} \\
E_{B A 2 i}^{*} & =E_{E E 2 i} e^{i \phi_{B A i}} \\
E_{B A 3 i}^{*} & =E_{E E 3 i} e^{i \phi_{B A i}} \\
G_{B A T}^{*} & =G_{E E T} e^{i \phi_{B A T}}
\end{aligned}
$$

\section{Evaluation of the Proposed Combined Back Analysis}

\subsection{Principle of the Comparison}

The procedure described in the previous section was used to determine the values of $f_{F i}, E_{F i}^{*}$ (norm and phase angle $\phi_{F i}$ ), $\left|v_{F i}^{*}\right|, \phi_{B A i}, E_{E E 1 i}, E_{E E 2 i}$ and $E_{E E 3 i}$ for the longitudinal and flexural modes of vibration and the values of $f_{F T}, G_{F T}^{*}$ (norm and phase angle $\phi_{F T}$ ), $\phi_{B A T}$ and $G_{E E T}$. All these values were calculated for the 10 considered temperatures. Table 2 details the name of the variables evaluated in the back

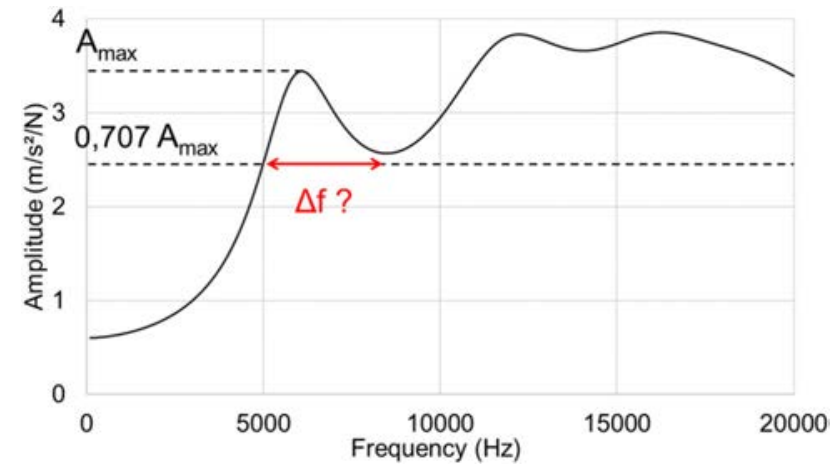

Fig. 8 Example of the half-power bandwidth method limitations at high temperatures where it was not possible to obtain $\Delta \mathrm{f}$ (numerical simulation for the longitudinal mode at $40{ }^{\circ} \mathrm{C}$ )

analysis in the different cases studied for clarity. Results are given in Tables 3, 4 and 5 in appendix.

For temperatures higher than $30^{\circ} \mathrm{C}$, it was not possible to estimate the phase angle of the material for the longitudinal and the torsional modes of vibration. This is due to too much damping in the material for such temperatures that made the estimation of the frequency bandwidth impossible with the half-power bandwidth method (see Fig. 8).

In order to evaluate the accuracy of the proposed combined back analysis, the different values obtained were compared. For the longitudinal and flexural modes of vibration, the norm and the phase angle of the 2S2P1D model complex modulus $E_{F i}^{*}$ obtained at the frequency $f_{F i}$ (Eq. 8) were compared to the norm and to the phase angle of the three complex moduli $E_{B A 1 i}^{*}, E_{B A 2 i}^{*}$ and $E_{B A 3 i}^{*}$ (Eqs. 12-14) obtained from the simplified back analysis. The three studied cases allow to consider the effect of the Poisson's ratio value. For the torsional mode of vibration, the norm and the phase angle of the 2S2P1D model complex shear modulus $G_{F T}^{*}$ obtained at the frequency $f_{F T}$ (Eq. 10) were compared to the norm and to the phase angle of the complex shear modulus $G_{B A T}^{*}$ (Eq. 15) obtained from the simplified back analysis.
Table 2 Summary of the

\begin{tabular}{|c|c|c|c|c|}
\hline $\begin{array}{l}\text { Mode of } \\
\text { vibration }\end{array}$ & Case studied & $\begin{array}{l}\text { Elastic equivalent } \\
\text { modulus }\end{array}$ & $\begin{array}{l}\text { Phase } \\
\text { angle }\end{array}$ & $\begin{array}{l}\text { Complex } \\
\text { modulus }\end{array}$ \\
\hline \multirow[t]{3}{*}{ Longitudinal } & $v_{1}=0.2$ & $\mathrm{E}_{\mathrm{EEIL}}$ & \multirow[t]{3}{*}{$\phi_{\mathrm{BAL}}$} & $\mathrm{E}_{\mathrm{BA} A \mathrm{~L}}^{*}$ \\
\hline & $v_{2}=\left|v_{2 \mathrm{~S} 2 \mathrm{P} 1 \mathrm{D}}^{*}\left(\mathrm{f}_{\mathrm{L}}\right)\right|$ & $\mathrm{E}_{\mathrm{EE} 2 \mathrm{~L}}$ & & $\mathrm{E}_{\mathrm{BA} 2 \mathrm{~L}}^{*}$ \\
\hline & $\nu_{3}=0.45$ & EEE3L $_{\text {E }}$ & & $\mathrm{E}_{\mathrm{BA} 3 \mathrm{~L}}^{*}$ \\
\hline \multirow[t]{3}{*}{ Flexural } & $v_{1}=0.2$ & EEEIF & \multirow[t]{3}{*}{$\phi_{\mathrm{BAF}}$} & $\mathrm{E}_{\mathrm{BA} 1 \mathrm{~F}}^{*}$ \\
\hline & $v_{2}=\left|v_{2 \mathrm{~S} 2 \mathrm{P} 1 \mathrm{D}}^{*}\left(\mathrm{f}_{\mathrm{F}}\right)\right|$ & $\mathrm{E}_{\mathrm{EE} 2 \mathrm{~F}}$ & & $\mathrm{E}_{\mathrm{BA} 2 \mathrm{~F}}^{*}$ \\
\hline & $v_{3}=0.45$ & $\mathrm{E}_{\mathrm{EE} 3 \mathrm{~F}}$ & & $\mathrm{E}_{\mathrm{BA} 3 \mathrm{~F}}^{*}$ \\
\hline Torsional & Independent of $\nu$ & $\mathrm{G}_{\mathrm{ET}}$ & $\phi_{\mathrm{BAT}}$ & $\mathrm{G}_{\mathrm{BAT}}^{*}$ \\
\hline
\end{tabular}
variables evaluated in the different cases studied for the back analysis 
Fig. 9 Relative difference between the elastic equivalent modulus obtained from the combined back analysis (Fig. 3) model value obtained at the first resonance frequency

(Eqs. 8-10) for the three considered modes of vibration Poisson's ratio values and the norm of the 2S2P1D and different considered
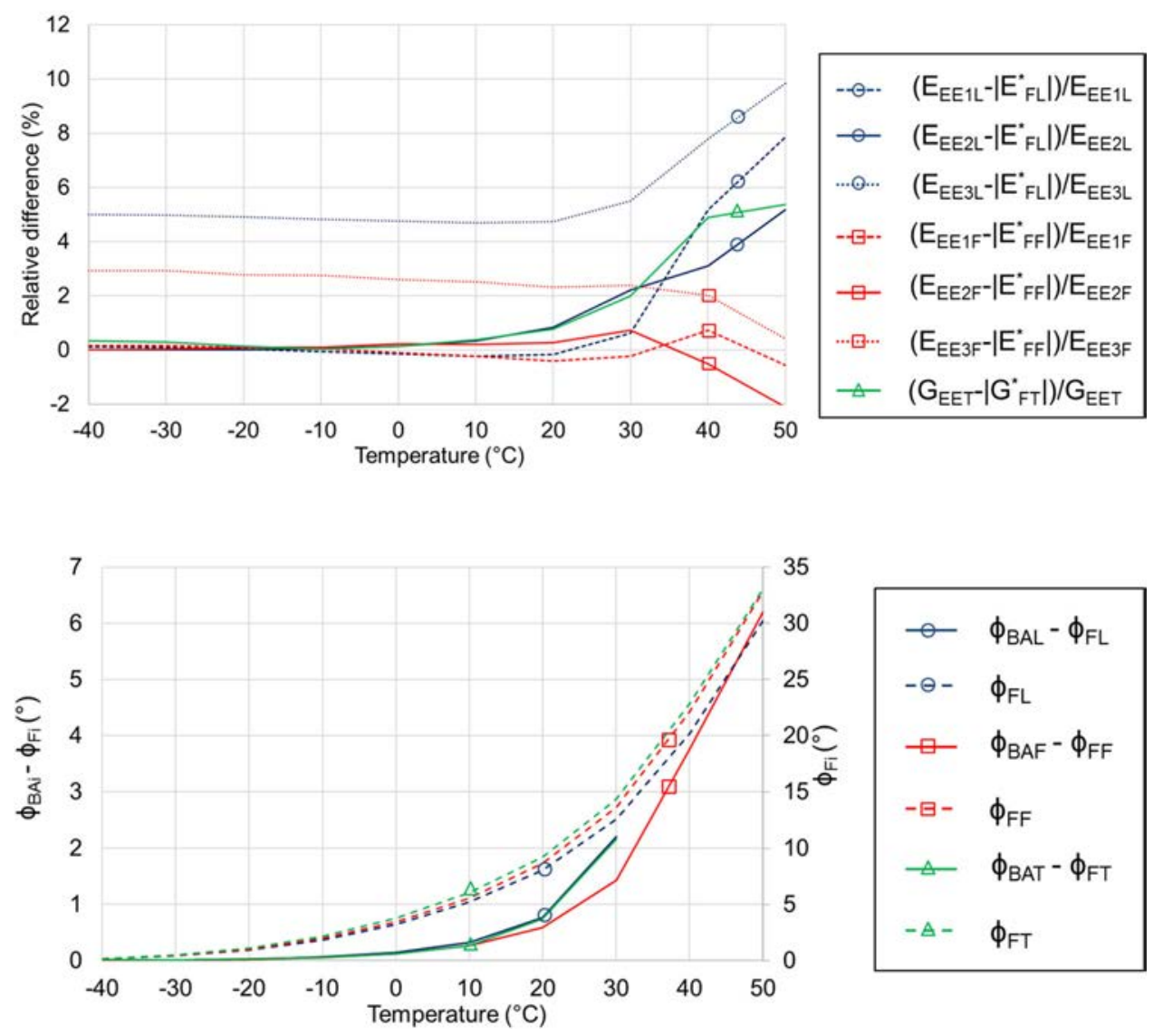

Fig. 10 2S2P1D model phase angle obtained at the first resonance frequency (Eqs. 8-10) (dot lines) and difference with the phase angle obtained from the combined back analysis (Fig. 3) (solid lines) for the three considered modes of vibration

Fig. 11 Complex modulus values obtained from the 2S2P1D model at the first resonance frequency of the longitudinal mode (Eq. 8) and values obtained from the proposed combined back analysis for the three considered values of Poisson's ratio (Fig. 3) for the longitudinal mode of vibration (Eqs. 12-14)

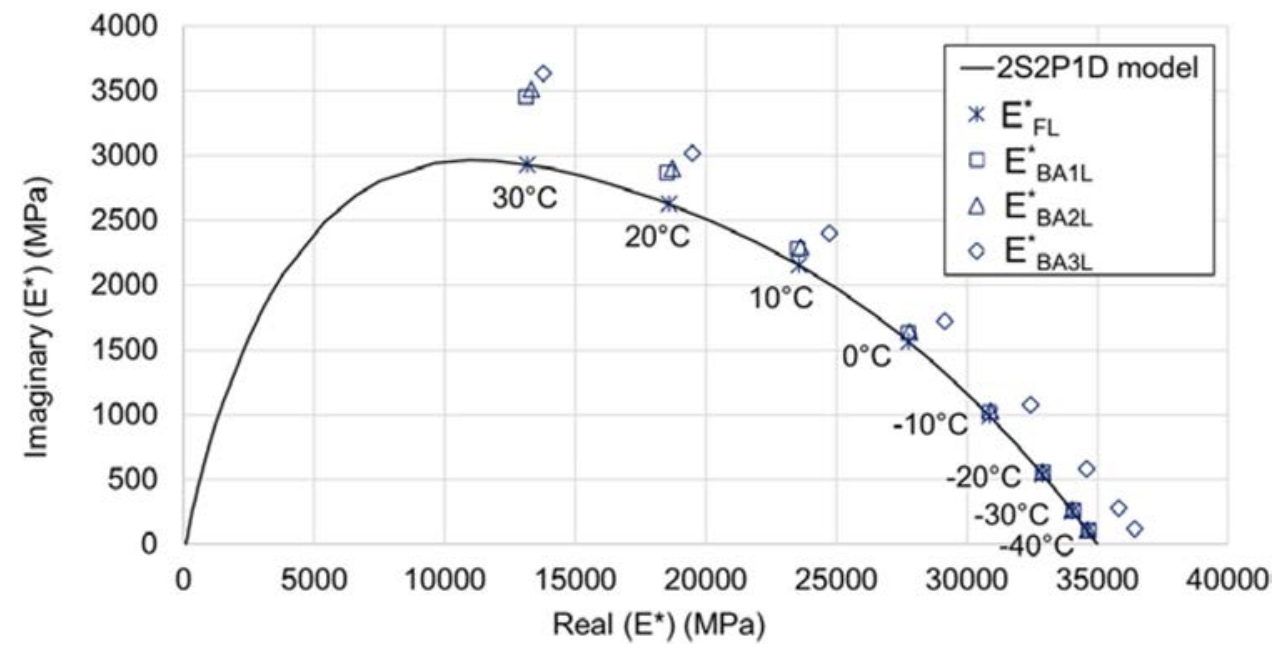

\subsection{Results and Discussion}

Figure 9 presents the relative difference between the elastic equivalent moduli $E_{E E 1 i}, E_{E E 2 i}$ and $E_{E E 3 i}$ and the norm of the complex modulus $\left|E_{F i}^{*}\right|$ calculated using the 2S2P1D model at the resonance frequencies $f_{F i}$. Lines with a circle (in blue) are for the longitudinal mode of vibration while lines with a square (in red) are for the flexural mode of vibration.
Also on Fig. 6, the relative difference between the elastic equivalent shear modulus $G_{E E T}$ and the norm of the complex shear modulus $\left|G_{F T}^{*}\right|$ calculated using 2S2P1D model at the frequency $f_{F T}$ is plotted (green line with a triangle). The solid lines on Fig. 6 clearly indicate that there is a good correlation between the elastic equivalent modulus $E_{E E 2 i}$ and the norm of the complex modulus from the 2S2P1D model $\left|E_{F i}^{*}\right|$ and also between the elastic equivalent shear modu- 
Fig. 12 Complex modulus values obtained from the 2S2P1D model at the first resonance frequency of the flexural mode (Eq. 8) and values obtained from the proposed combined back analysis for the three considered values of Poisson's ratio (Fig. 3) for the flexural mode of vibration (Eqs. 12-14)
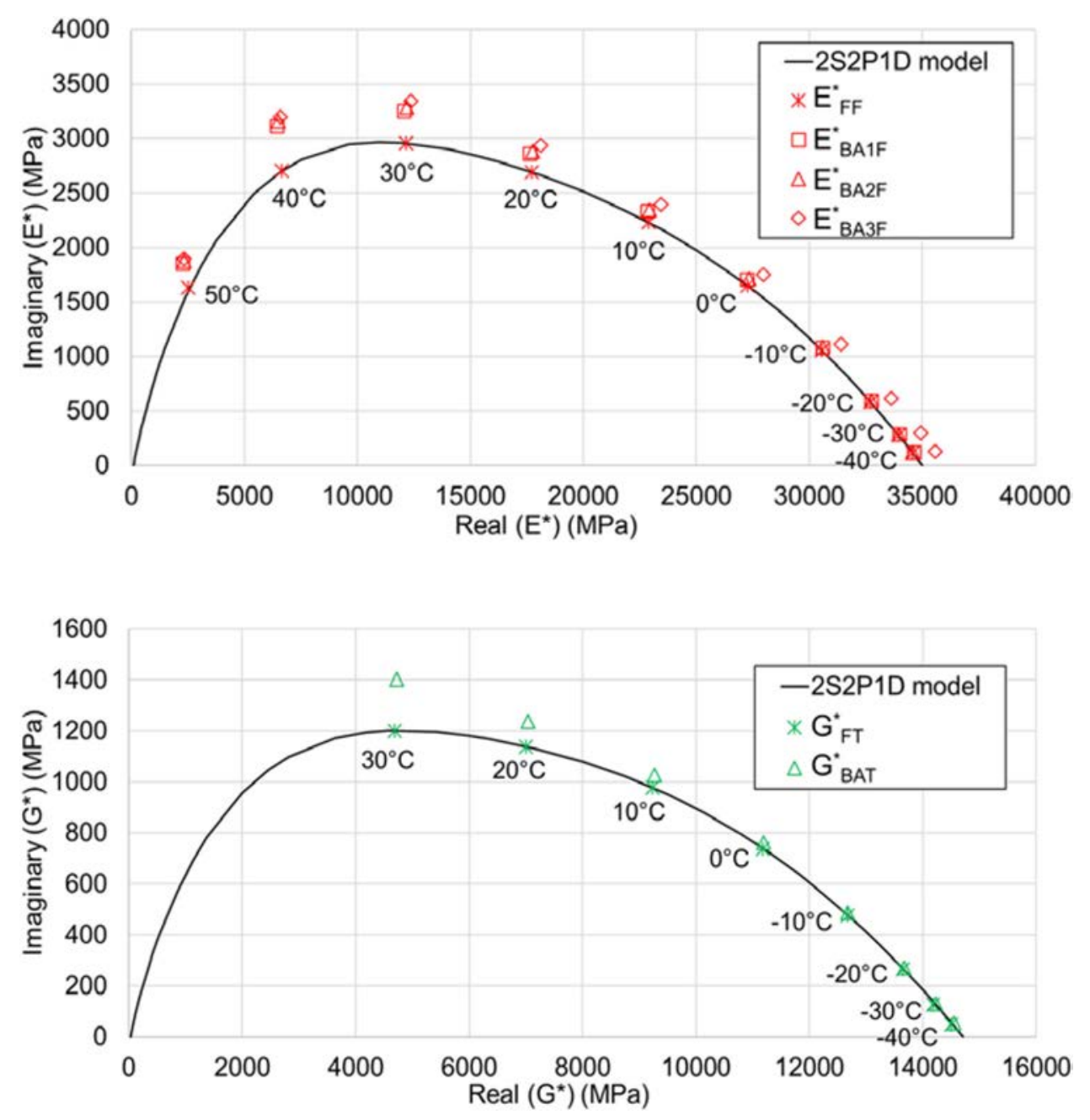

Fig. 13 Complex shear modulus values obtained from the 2S2P1D model at the first resonance frequency of the torsional mode (Eq. 10) and values obtained from the proposed combined back analysis (figure 3) for the torsional mode of vibration (Eq. 15) lus and the norm of the complex shear modulus from the 2S2P1D model $\left|G_{F T}^{*}\right|$. This is particularly true for temperatures below $30{ }^{\circ} \mathrm{C}$ with a maximum relative difference of less than $2.5 \%$. At higher temperatures, the error increases for the three modes of vibration but it remains less than $6 \%$. It can also be seen on Fig. 6 that the Poisson's ratio value has an impact on the calculation of the elastic equivalent moduli. The relative difference is clearly higher for $E_{E E 3 i}$ (around $3 \%$ for the flexural mode and around $5 \%$ for the longitudinal mode) that is calculated for a Poisson's ratio of 0.45 , a value away from the two other studied cases. Nevertheless, the impact of the Poisson's ratio value remains limited.

Figure 10 presents the phase angle $\phi_{F i}$ given by the 2S2P1D model at the frequency $f_{F i}$ (dot lines) and also the difference in degree $\left({ }^{\circ}\right)$ with the combined back analysis phase angle $\phi_{B A i}$ for the three considered modes of vibration. Figure 4 shows a good agreement between the back analysis phase angle $\phi_{B A i}$ and the 2S2P1D model phase angle $\phi_{F i}$ with a maximum difference of around $2^{\circ}$ at $30^{\circ} \mathrm{C}$ for the longitudinal and torsional modes. For the flexural mode, it can be seen that the difference significantly increases for temperatures above $30{ }^{\circ} \mathrm{C}$ to reach $6^{\circ}$ at $50{ }^{\circ} \mathrm{C}$. This proves the inaccuracy of the half-power bandwidth method at high temperature: when there is too much damping in the material, the damping is overestimated and so is the phase angle.

For the complex modulus or complex shear modulus as well as for the phase angle, differences between values obtained from the 2S2P1D model at the first resonance frequency and values obtained with the combined back analysis increase with the temperature but remain limited. Figures 11, 12 and 13 present the different complex moduli in a ColeCole diagram for the three considered modes of vibration and the three different values of Poisson's ratio. On Figs. 7 and 8 corresponding to the longitudinal mode and to the flexural mode respectively, it can be seen that the values of $E_{B A 1 i}^{*}$ or $E_{B A 2 i}^{*}$ and $E_{F i}^{*}$ are rather close, especially at low temperatures. $E_{B A 3 i}^{*}$ does not fit so well with $E_{F i}^{*}$. It is due to the effect of the Poisson's ratio value as already noted on Fig. 6. Figure 9 corresponding to the torsional mode shows a good agreement between $G_{B A T}^{*}$ and $G_{F T}^{*}$, especially for the low temperatures. All the observations from Figs. 11, 12 and 13 confirm the conclusions already raised from Figs. 9 and 10. 


\section{Conclusion}

The proposed simplified back analysis proposed in this paper gives good results, both for the norm of the complex modulus and for the phase angle at low and intermediate temperatures. Slight differences appear for temperatures higher than $20^{\circ} \mathrm{C}$. They are mostly due to an overestimation of the phase angle. Therefore, the half-power bandwidth method used to determine the phase angle is not adapted for high temperatures because it overestimates the damping of the materials. The influence of the Poisson's ratio value was also studied. It was found that it has a limited impact on the calculation of the elastic equivalent modulus (less than 5\%). The observations and findings from this study demonstrate that for temperatures below $20^{\circ} \mathrm{C}$, a simple assumption on constant real value for Poisson's ratio and the proposed simplified combined back analysis are enough to analyse accurately impact loadings in order to obtain the behaviour of asphalt mixes.. This is very interesting to obtain quickly and accurately the LVE properties at high frequencies. However, the limitation with the temperature gives only access to a small part of the master curve that can be estimated with this method. In addition, for higher temperatures corresponding to values of the phase angle higher than about $15^{\circ}$, the method does not provide a good evaluation of the phase angle. Therefore, for temperatures higher than $30{ }^{\circ} \mathrm{C}$ corresponding to phase angle of above $15^{\circ}$ the half-power bandwidth could not be used because of too much damping in the material. These numerical results need to be checked and validated in further studies using laboratory measurements.

\section{Appendix}

Table 3 Results of the numerical simulations and the combined back analysis (longitudinal mode)

\begin{tabular}{|c|c|c|c|c|c|c|c|c|}
\hline \multirow[t]{2}{*}{$T\left({ }^{\circ} \mathrm{C}\right)$} & \multicolumn{3}{|l|}{ 2S2P1D model } & \multicolumn{5}{|c|}{ Combined back analysis } \\
\hline & $\left|E_{F L}^{*}\right|(M P a)$ & $\phi_{F L}\left(^{\circ}\right)$ & $\left|v_{F L}^{*}\right|$ & $f_{F L}(H z)$ & $\phi_{B A L}\left(^{\circ}\right)$ & $E_{E E 1 L}(M P a)$ & $E_{E E 2 L}(M P a)$ & $E_{E E 3 L}(M P a)$ \\
\hline-40 & 34,602 & 0.178 & 0.191 & 12,320 & 0.186 & 34,645 & 34,605 & 34,425 \\
\hline-30 & 34,029 & 0.434 & 0.194 & 12,216 & 0.450 & 34,060 & 34,030 & 35,810 \\
\hline-20 & 32,890 & 0.943 & 0.198 & 12,006 & 0.969 & 32,900 & 32,890 & 34,585 \\
\hline-10 & 30,891 & 1.837 & 0.206 & 11,631 & 1.901 & 30,870 & 30,910 & 32,460 \\
\hline 0 & 27,813 & 3.232 & 0.219 & 11,031 & 3.372 & 27,775 & 27,850 & 29,200 \\
\hline 10 & 23,675 & 5.219 & 0.238 & 10,173 & 5.542 & 23,620 & 23,755 & 24,840 \\
\hline 20 & 18,770 & 8.049 & 0.264 & 9062 & 8.813 & 18,740 & 18,930 & 19,705 \\
\hline 30 & 13,475 & 12.575 & 0.294 & 7708 & 14.782 & 13,560 & 13,780 & 14,260 \\
\hline 40 & 8179 & 20.188 & 0.327 & 6080 & $x$ & 8440 & 8625 & 8870 \\
\hline 50 & 3746 & 30.621 & 0.362 & 4160 & $x$ & 3950 & 4065 & 4155 \\
\hline
\end{tabular}

Table 4 Results of the numerical simulations and the combined back analysis (flexural mode)

\begin{tabular}{|c|c|c|c|c|c|c|c|c|}
\hline \multirow[t]{2}{*}{$T\left({ }^{\circ} \mathrm{C}\right)$} & \multicolumn{3}{|l|}{ 2S2P1D model } & \multicolumn{5}{|c|}{ Combined back analysis } \\
\hline & $\left|E_{F F}^{*}\right|(M P a)$ & $\phi_{F F}\left(^{\circ}\right)$ & ||$v_{F F}^{*} \mid$ & $\overline{f_{F F}(H z)}$ & $\phi_{B A F}\left(^{\circ}\right)$ & $E_{E E 1 F}(M P a)$ & $E_{E E 2 F}(M P a)$ & $E_{E E 3 F}(M P a)$ \\
\hline-40 & 34,569 & 0.193 & 0.192 & 7619 & 0.203 & 34,625 & 34,575 & 35,580 \\
\hline-30 & 33,948 & 0.47 & 0.194 & 7549 & 0.486 & 34,000 & 33,960 & 34,940 \\
\hline-20 & 32,720 & 1.019 & 0.199 & 7410 & 1.044 & 32,750 & 32,750 & 33,630 \\
\hline-10 & 30,580 & 1.977 & 0.207 & 7161 & 2.031 & 30,595 & 30,610 & 31,420 \\
\hline 0 & 27,317 & 3.461 & 0.221 & 6764 & 3.587 & 27,290 & 27,380 & 28,030 \\
\hline 10 & 22,991 & 5.571 & 0.242 & 6201 & 5.838 & 22,935 & 23,040 & 23,570 \\
\hline 20 & 17,933 & 8.63 & 0.268 & 5472 & 9.218 & 17,860 & 17,980 & 18,350 \\
\hline 30 & 12,518 & 13.671 & 0.3 & 4573 & 15.101 & 12,490 & 12,610 & 12,815 \\
\hline 40 & 7177 & 22.12 & 0.334 & 3460 & 25.880 & 7140 & 7230 & 7325 \\
\hline 50 & 3002 & 32.832 & 0.37 & 2220 & 39.036 & 2940 & 2985 & 3015 \\
\hline
\end{tabular}


Table 5 Results of the numerical simulations and the combined back analysis (torsional mode)

\begin{tabular}{|c|c|c|c|c|c|}
\hline \multirow[t]{2}{*}{$T\left({ }^{\circ} \mathrm{C}\right)$} & \multicolumn{2}{|l|}{ 2S2P1D model } & \multicolumn{3}{|c|}{ Combined back analysis } \\
\hline & $\left|G_{F T}^{*}\right|(M P a)$ & $\phi_{F T}\left({ }^{\circ}\right)$ & $f_{F T}(H z)$ & $\phi_{B A T}\left(^{\circ}\right)$ & $G_{E E T}(M P a)$ \\
\hline-40 & 14,502 & 0.192 & 8033 & 0.214 & 14,550 \\
\hline-30 & 14,184 & 0.514 & 7954 & 0.526 & 14,225 \\
\hline-20 & 13,653 & 1.107 & 7795 & 1.139 & 13,670 \\
\hline-10 & 12,681 & 2.152 & 7512 & 2.203 & 12,685 \\
\hline 0 & 11,205 & 3.769 & 7065 & 3.887 & 11,220 \\
\hline 10 & 9284 & 6.051 & 6437 & 6.321 & 9320 \\
\hline 20 & 7090 & 9.227 & 5639 & 9.967 & 7145 \\
\hline 30 & 4834 & 14.371 & 4684 & 16.540 & 4930 \\
\hline 40 & 2711 & 22.839 & 3560 & $\times$ & 2850 \\
\hline 50 & 1107 & 33.091 & 2280 & $x$ & 1170 \\
\hline
\end{tabular}

\section{References}

1. Migliori, A., Sarrao, J., Visscher, W., Bell, T., Ming, L., Fisk, Z., Leisure, R.: Resonant ultrasound spectroscopic techniques for measurement of the elastic moduli of solids. Physica B 183, 1-24 (1993)

2. Di Benedetto, H., Sauzéat, C., Sohm, J.: Stiffness of bituminous mixtures using ultrasonic wave propagation. Road Mater. Pavement Des. 10(4), 789-814 (2009). https://doi.org/10.1080/14680629. 2009.9690227

3. Mounier, D., Di Benedetto, H., Sauzéat, C.: Determination of bituminous mixtures linear properties using ultrasonic wave propagation. Constr. Build. Mater. 36, 638-647 (2012). https://doi.org/ 10.1061/j.conbuildmat.2012.04.136

4. Norambuena-Contreras, J., Castro-Fresno, D., Vega-Zamanillo, A., Celay, M., Lombillo-Vozmediano, I.: Dynamic modulus of asphalt mixture by ultrasonic direct test. NDT E Int. 43(7), 629-634 (2010)

5. Kweon, G., Kim, Y.R.: Determination of the complex modulus of asphalt concrete using the impact resonance test. J. Transp. Res. Board 1970, 151-160 (2006)

6. Whitmoyer, S.L., Kim, Y.R.: Determining asphalt concrete properties via the impact resonant method. J. Test. Eval. 22(2), 139-148 (1994)

7. Tavassoti-Kheiry, P., Boz, I., Solaimanian, M., Qiu, T.: Evaluation of nondestructiveness of resonant column testing for characterization of asphalt concrete properties. J. Test. Eval. 46(2), 631-640 (2018)

8. ASTM: C215-08. Standard Test Method for Fundamental Transverse. ASCE, Longitudinal and Torsional Frequencies of Concrete Specimens, West Conshoshocken (2008)

9. Gudmarsson, A., Ryden, N., Birgisson, B.: Application of resonant acoustic spectroscopy to asphalt concrete beams for determination of the dynamic modulus. Mater. Struct. 45, 1903-1913 (2012). https://doi.org/10.1617/s11527-012-9877-3

10. Ryden, N.: Resonant frequency testing of cylindrical asphalt samples. Eur. J. Environ. Civil Eng. 15, 587-600 (2011)

11. Mangiafico, S., Di Benedetto, H., Sauzéat, C., et al.: Influence of reclaimed asphalt pavement content on complex modulus of asphalt binder blends and corresponding mixes: experimental results and modelling. Road Mater. Pavement Des. 14(S1), 132-148 (2013)

12. Mangiafico, S., Di Benedetto, H., Sauzéat, C., et al.: Statistical analysis of the influence of RAP and mix composition on viscoelastic and fatigue properties of asphalt mixes. Mater. Struct. 48(4), 11871205 (2015)

13. Nguyen, H.P., Di Benedetto, H., Sauzéat, C., et al.: Reclaimed asphalt pavement and additives' influence on $3 \mathrm{D}$ linear behavior of warm mix asphalts. Road Mater. Pavement Des. 16(3), 569-591 (2015)

14. Nguyen, H.P., Di Benedetto, H., Sauzéat, C., et al.: Analysis and modeling of 3D complex modulus tests on hot and warm bituminous mixtures. Mech. Time-depend. Mater. 19(2), 167-186 (2015)

15. Nguyen, Q.T.: Comportement thermomécanique des enrobés bitumineux sous sollicitations cycliques dans les domaines linéaires et non-linéaire. Ph.D. Thesis, University of Lyon, ENTPE, France (2011)

16. Nguyen, Q.T., Di Benedetto, H., Sauzéat, C., Tapsoba, N.: Time-temperature superposition principle validation for bituminous mixes in the linear and nonlinear domain. ASCE J. Mater. Civil Eng. 25(9), 1181-1188 (2013). https://doi.org/10.1061/ (ASCE)MT.1943-5533.0000658

17. Viet, P.C., Di Benedetto, H., Sauzéat, C., Lesueur, D., Pouget, S., Olard, F., Dupriet, S.: Complex modulus and fatigue resistance of bituminous mixtures containing hydrated lime. Constr. Build. Mater. 139, 24-33 (2017). https://doi.org/10.1016/j.conbuildmat. 2017.02.042

18. Gudmarsson, A., Ryden, N., Di Benedetto, H., Sauzéat, C., Tapsoba, N., Birgisson, B.: Comparing linear viscoelastic properties of asphalt concrete measured by laboratory seismic and tensioncompression tests. J. Nondestr. Eval. 33, 571-582 (2014). https:// doi.org/10.1007/s10921-014-0253-9

19. Gudmarsson, A., Ryden, N., Di Benedetto, H., Sauzéat, C.: Complex modulus and complex Poisson's ratio from cyclic and dynamic modal testing of asphalt concrete. Constr. Build. Mater. 88, 20-31 (2015). https://doi.org/10.1016/j.conbuildmat.2015.04.007

20. Di Benedetto, H., Olard, F., Sauzéat, C., Delaporte, B.: Linear viscoelastic behavior of bituminous materials: from binders to mixtures. Road Mater. Pavement Des. 5(S1), 163-202 (2004). https:// doi.org/10.1080/14680629.2004.9689992

21. Olard, F., Di Benedetto, H.: General 2S2P1D model and relation between the linear viscoelastic behaviors of bituminous binders and mixes. Road Mater. Pavement Des. 4(2), 185-224 (2003). https:// doi.org/10.1080/14680629.2003.9689946

22. Tiouajni, S., Di Benedetto, H., Sauzéat, C., Pouget, S.: Approximation of linear viscoelastic model in the 3 dimensional case with mechanical analogues of finite size - application to bituminous materials. Road Mater. Pavement Des. 12(4), 897-930 (2011). https://doi.org/10.1080/14680629.2011.9713899

23. Gourdon, E., Sauzéat, C., Di Benedetto, H., Bilodeau, K.: Sevenparameter linear viscoelastic model applied to acoustical damping materials. J. Vib. Acoust. 137(6), 061003 (2015). https://doi.org/ $10.1115 / 1.4030719$ 
24. Nguyen, H.M., Pouget, S., Di Benedetto, H., Sauzéat, C.: Timetemperature superposition principle for bituminous mixtures. Eur. J. Environ. Civil Eng. 13(9), 1095-1107 (2009). https://doi.org/10. 3166/ejece.13.1095-1107

25. Nguyen, M.L., Sauzéat, C., Di Benedetto, H., Tapsoba, N.: Validation of the time-temperature superposition principle for crack propagation in bituminous mixtures. Mater. Struct. 46(7), 10751087 (2013). https://doi.org/10.1617/s11527-012-9954-7

26. Ferry, J.D.: Viscoelastic Properties of Polymers, 3rd edn. Wiley, New York (1980)

27. Di Benedetto, H., Delaporte, B., Sauzéat, C.: Three dimensional linear behavior of bituminous materials: experiments and modeling. ASCE Int. J. Geomech. 7(2), 149-157 (2007). https://doi.org/ 10.1061/(ASCE)1532-3641(2007)7:2(149)

28. Cannone Falchetto, A., Moon, K.-H., Lee, C.B., Wistuba, M.P.: Correlation of low temperature fracture and strength properties between SCB and IDT tests using a simple 2D FEM approach. Road Mater. Pavement Des. 18(sup2), 329-338 (2017). https://doi. org/10.1080/14680629.2017.1304258

29. Cho, Y.S., Lin, F.B.: Spectral analysis of surface waves in single and multi-layer slabs with finite thickness using finite element modelling. NDT E Int. 38(3), 195-202 (2005)
30. Taherkhani, H., Jalali, M.: Investigating the performance of geosynthetic-reinforced asphaltic pavement under various axle loads using finite-element method. Road Mater. Pavement Des. (2016). https://doi.org/10.1080/14680629.2016.1201525

31. Yesuf, G.Y., Hoff, I.: Finite element modelling for prediction of permanent strains in fine-grained subgrade soils. Road Mater. Pavement Des. 16(2), 392-404 (2015). https://doi.org/10.1080/ 14680629.2015.1013053

32. You, L., Yan, K., Hu, Y., Liu, J., Ge, D.: Spectral element method for dynamic response of transversely isotropic asphalt pavement under impact load. Road Mater. Pavement Des. (2016). https://doi. org/10.1080/14680629.2016.1230513

33. Madsen, S.S., Levenberg, E.: Dynamic backcalculation with different load-time histories. Road Mater. Pavement Des. (2017). https:// doi.org/10.1080/14680629.2017.1307263

34. Riccardi, C., Cannone Falchetto, A., Leandro, P., Losa, M., Wistuba, M.: A novel back-calculation approach for determining the rheological properties of RAP binder. Road Mater. Pavement Des. 18(sup1), 359-381 (2016). https://doi.org/10.1080/ 14680629.2016.1266778

35. Clough, R.W., Penzien, J.: Dynamics of Structures, 2nd edn. McGraw-Hill Inc, New-York (1993) 